\title{
ANISOTROPIC PHASE-FIELD DESCRIPTION OF VON KÁRMÁN BEAMS
}

\author{
J. Schmidt*, T. Janda**, J. Zeman***, A. Zemanová ${ }^{\dagger}$, M. Šejnoha ${ }^{\ddagger}$
}

\begin{abstract}
For brittle materials with complex crack topology, discrete crack approach suffers from implementation complexity. The phase-field formulation can overcome this issue. In this paper, using phase-field approach for brittle beams and plates is reviewed. We assume that the damage appears only in tension, meanwhile in compression the material is intact. Accordingly, the damage field for these cases is only scalar field and each FEM node has only one extra unknown variable. Moreover in staggered approach, calculation is divided into two linear forms and inner iterative procedure is not needed for Mindlin beams. It does not hold for von Kármán large deflection formulation as presented at the end of the text.
\end{abstract}

\section{Keywords: phase-field, brittle fracture, Mindlin beam, von Kármán, large deflection}

\section{Introduction}

This paper provides a theoretical background for crack initialization and propagation model for multilayer laminated glass beams by investigating damage evolution in a single glass layer subjected to deflection. The principles of variational phase-field formulation and the Von Kármán member for large deflections are exploited. In context of beam elements, we consider damage $d$ being 1D scalar field depending on position variable $x$ only. The phase-field approach to damage on beams is derived in footsteps of Kiendl (2016).

\section{1D Phase-field formulation of brittle fracture}

The solution of crack propagation can be governed by variational formulation. The energy functional, which is being minimized, is given in the Griffith sense by

$$
\mathcal{E}(\varepsilon, \Gamma)=\int_{\Omega} \Psi(\varepsilon) d \Omega+G_{c} \int_{\Gamma} d \Gamma
$$

where $\Omega$ is the problem domain, $\Gamma \subset \Omega$ is the internal discontinuity boundary, $\Psi$ is the elastic energy density dependent on an actual strain $\varepsilon$ and $G_{c}$ is energy dissipated when unit of fracture surface is created. The Griffith approach assumes pefrectly sharp crack and non-smooth course of damage, while the phasefield formulation approximates smeared damage field over domain $(-\infty, \infty)$. Figure 1 represents such that distribution of damage field $d$ around the crack at point $x=0$. Value $d=0$ stands for unbroken state and $d=1$ for fully broken state. The damage field approximation is given by an exponential function proposed by Miehe (2010)

\footnotetext{
Bc. Jaroslav Schmidt: Faculty of Civil Engineering, Czech Technical University in Prague, Thákurova 7, 166 29, Prague, CZ, jarasit@gmail.com

** Ing. Tomáš Janda, Ph.D.: Faculty of Civil Engineering, Czech Technical University in Prague, Thákurova 7, 166 29, Prague, CZ, tomas.janda@fsv.cvut.cz

*** Doc. Ing. Jan Zeman, Ph.D.: Faculty of Civil Engineering, Czech Technical University in Prague, Thákurova 7, 16629 , Prague, CZ, Jan.Zeman@ @ cvut.cz

† Ing. Alena Zemanová, Ph.D.: Faculty of Civil Engineering, Czech Technical University in Prague, Thákurova 7, 166 29, Prague, CZ, zemanova.alena@gmail.com

‡ Prof. Ing. Michal Šejnoha, Ph.D., DSc.: Faculty of Civil Engineering, Czech Technical University in Prague, Thákurova 7 , 166 29, Prague, CZ, sejnom@fsv.cvut.cz
} 


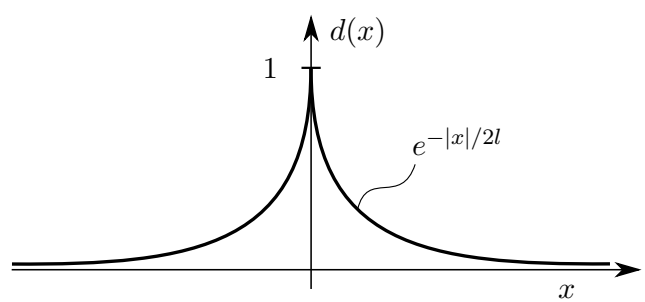

Fig. 1: The distribution of damage parameter $d$ around crack at $x=0$

$$
d(x)=e^{-|x| / 2 l_{c r}},
$$

where $l_{c r}$ is the characteristic length or length of the process zone. We can observe that equation (2) is the solution of boundary value problem

$$
d-4 l_{c r}^{2} d^{\prime \prime}(x)=0, \quad d(0)=1, \quad d( \pm \infty)=0 .
$$

According to the Hamilton's principle this equation is given by Euler-Lagrange equation of action integral

$$
\mathcal{S}(d)=\int_{\Omega} \frac{1}{4} d^{2}+l_{c r}^{2}\left|d^{\prime}(x)\right|^{2} d \Omega .
$$

It can be shown that the integrand divided by $l_{c r}$ represents crack density and the action integral represent volume approximation of crack surface, so

$$
\Gamma=\int_{\Gamma} d \Gamma \approx \frac{1}{l_{c r}} \mathcal{S}(d)
$$

and the governing functional becomes

$$
\mathcal{E}(\varepsilon, d)=\int_{\Omega} \Psi(\varepsilon) d \Omega+G_{c} \int_{\Omega} \frac{1}{4 l_{c r}} d^{2}+l_{c r}\left|d^{\prime}(x)\right|^{2} d \Omega=\int_{\Omega} \mathcal{L} d \Omega .
$$

For realistic modeling of damage it is convenient decompose the strain tensor into the tensile and compressive parts. Similarly, we decompose the elastic density function

$$
\Psi(\varepsilon, d)=g(s) \Psi^{+}(\varepsilon)+\Psi^{-}(\varepsilon),
$$

where only the tensile part is degraded by the degradation function $g(s)$. We employ the degradation function from Miehe (2010)

$$
g(s)=(1-\eta) s^{2}+\eta,
$$

where we use rather the damage parameter $s(x)=1-d(x)$ than $d(x)$. The quadratic form of equation (8) provides an lower bound of the phase-field $s$. The parameter $\eta$ represents residual stiffness factor, which ensures well-posed problem. Now the Hamilton's principle gives us the governing equations of the system by minimization of the phase field $s$ and deformation $\varepsilon$ via Euler-Lagrange equations which hold

$$
\begin{gathered}
\operatorname{div}\left(g(s) \frac{\partial \Psi^{+}(\varepsilon)}{\partial \varepsilon}+\frac{\partial \Psi^{-}(\varepsilon)}{\partial \varepsilon}\right)=\mathbf{0}, \\
\left(\frac{4 l_{c r}(1-\eta) \mathcal{H}}{G_{c}}+1\right) s-4 l_{c r}^{2} s^{\prime \prime}(x)=1 .
\end{gathered}
$$

The history variable $\mathcal{H}$ is given by $\mathcal{H}=\max \Psi^{+}(\tau)$ across all times $\tau \in\langle 0, t\rangle$. The variable $\mathcal{H}$ ensures irreversibility of damage.

\section{Phase-field fracture for beams}

For beams formulation we employ the Mindlin beam theory, which gives to us the following energy density function

$$
\Psi(\varepsilon)=\frac{1}{2} \varepsilon_{i j} \sigma_{i j}=\frac{1}{2}\left(\varepsilon_{x} \sigma_{x}+\gamma_{x z} \tau_{x z}\right)=\frac{1}{2}\left(E \varepsilon_{x}^{2}+G \gamma_{x z}^{2}\right),
$$



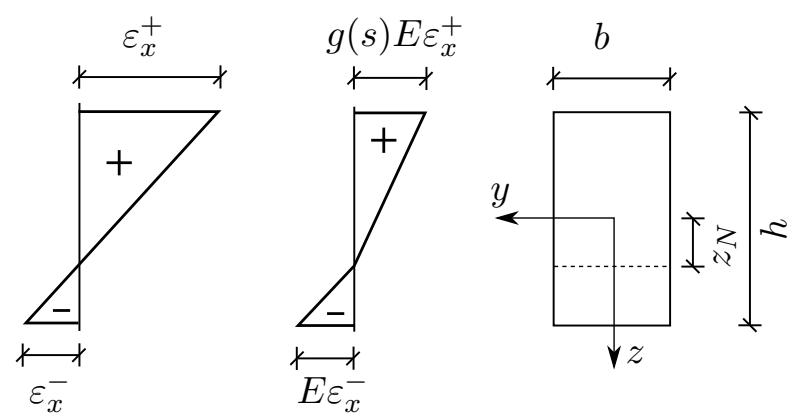

Fig. 2: Course of strain and stress on cross section with tensile degradation

and the normal strain of cross section in $x$ direction of the beam centerline

$$
\varepsilon_{x}(x)=u_{0}^{\prime}(x)+\varphi^{\prime}(x) z,
$$

where $u_{0}(x)$ is the centerline longitudinal displacement caused by a membrane forces and $\varphi(x)$ is a sectional rotation. The height $z_{N}$ where $\varepsilon_{x}=0$ splits the cross-section on two parts, which one is in the compression and other is in tension. If we define such split, we can tensil part reduce by the degradation function $g(s)$. This is illustrated in figure 2, where first picture represents the distribution of strain along height and the second picture shows the evolution of normal stress along height. The last picture defines variables $b, h$, the position of neutral axis $z_{N}$ and axis direction on the cross section. The stored elastic energy then reads

$$
\int_{\Omega} \Psi(\varepsilon) d \Omega=\frac{b}{2} \int_{0}^{L} \xi^{ \pm}\left(-\frac{h}{2}, z_{N}\right) \int_{-h / 2}^{z_{N}} E \varepsilon_{x}^{2}+G \gamma_{x z}^{2} d z+\xi^{ \pm}\left(z_{N}, \frac{h}{2}\right) \int_{z_{N}}^{h / 2} E \varepsilon_{x}^{2}+G \gamma_{x z}^{2} d z d x
$$

where $\xi(a, b)$ acquires two values

$$
\xi^{ \pm}(a, b)= \begin{cases}g(s) & \text { if cross-section between } z=a \text { and } z=b \text { is in tension } \\ 1 & \text { otherwise }\end{cases}
$$

and the neutral axis position is derived from the the equation (12) and holds

$$
z_{N}(x)=-\frac{u_{0}^{\prime}(x)}{\varphi^{\prime}(x)}
$$

The equation (13) and the values of $\xi^{ \pm}$define division of the energy density function $\Psi$ to tensile and compressive parts. Members, where the degradation function $g(s)$ appears, belongs to the tension part $\Psi^{+}$ and members without the degradation function belongs to $\Psi^{-}$. We note that this formulation only applies to small shear.

\section{FEM formulation of beams}

The finite element formulation is derived in standard way. Unknown fields are approximated by matrix of base functions $\mathbf{N}$ and the gradients are approximated by geometric matrix $\mathbf{B}$. For the generalized displacement field $\boldsymbol{u}^{e}(x)$ and the damage field $s^{e}(x)$ on one finite element $e$ it reads

$$
\begin{gathered}
\boldsymbol{u}^{e}(x) \approx \mathbf{N}_{u}^{e}(x) \boldsymbol{r}_{u}^{e}, \quad \boldsymbol{\varepsilon}^{e} \approx \frac{\partial \boldsymbol{u}^{e}(x)}{\partial x} \approx \mathbf{B}_{u}^{e}(x) \boldsymbol{r}_{u}^{e}, \\
s^{e}(x) \approx \mathbf{N}_{s}^{e}(x) \boldsymbol{r}_{s}^{e}, \quad s^{\prime}(x) \approx \mathbf{B}_{s}^{e}(x) \boldsymbol{r}_{s}^{e},
\end{gathered}
$$

where $r^{e}$ are vectors of nodal values. With these base functions, the weak form of governing equations (9)-(10) on element $e$ have the following discrete form

$$
\left((1-\eta)\left(\boldsymbol{r}_{s}^{e}\right)^{\mathrm{T}} \mathbf{M}_{2} \boldsymbol{r}_{s}^{e}+\eta\right) \frac{\partial \Psi^{+}\left(\boldsymbol{r}_{u}^{e}\right)}{\partial \boldsymbol{r}_{u}^{e}}+\frac{\partial \Psi^{-}\left(\boldsymbol{r}_{u}^{e}\right)}{\partial \boldsymbol{r}_{u}^{e}}=\mathbf{0}
$$




$$
\left(\frac{4 l_{c r}(1-\eta) \mathcal{H}}{b h G_{c}}+1\right) \mathbf{M}_{2} \boldsymbol{r}_{s}^{e}+4 l_{c r}^{2} \mathbf{M}_{1} \boldsymbol{r}_{s}^{e}=\int_{L^{e}}\left(\mathbf{N}_{s}^{e}\right)^{\mathrm{T}} d L^{e},
$$

where matrices $\mathbf{M}_{1}$ and $\mathbf{M}_{2}$ are defined as

$$
\mathbf{M}_{1}=\int_{L^{e}}\left(\mathbf{B}^{e}\right)^{\mathrm{T}} \mathbf{B}^{e} d L^{e}, \quad \mathbf{M}_{2}=\int_{L^{e}}\left(\mathbf{N}^{e}\right)^{\mathrm{T}} \mathbf{N}^{e} d L^{e} .
$$

The equations (18)-(19) for the case of linear elasticity can be both expressed in linear form

$$
\mathbf{K}_{u} \boldsymbol{r}_{u}=\boldsymbol{R}_{u}, \quad \mathbf{K}_{s} \boldsymbol{r}_{s}=\boldsymbol{R}_{s},
$$

where $\boldsymbol{R}_{u}$ is the vector of external nodal forces and $\boldsymbol{R}_{s}$ is the right hand side of equation (19). The forms of stiffness matrix $\mathbf{K}_{u}$ and the form of system matrix $\mathbf{K}_{s}$ are obvious from the equations (18)-(19). In the stagger approach, these matrix equations are independent. If we solve ${ }^{k} \boldsymbol{r}_{u}$ for $k$-th iterative step we assume fixed ${ }^{k-1} \boldsymbol{r}_{s}$ from history and vice versa for the field ${ }^{k} \boldsymbol{r}_{s}$ is ${ }^{k} \boldsymbol{r}_{u}$ fixed. For given external loads we iterate through $k$ until both fields are stationary.

\section{Von Kármán formulation}

To consider large deflection and small strains we add the Von Kármán term into the expression of normal strain, which modifies only equation (12) to the shape

$$
\varepsilon_{x}(x)=u_{0}^{\prime}(x)+\varphi^{\prime}(x) z+\frac{1}{2}\left(w^{\prime}(x)\right)^{2},
$$

where $w(x)$ is deflection in $z$ direction. It complicates situation, because the discrete form (18) can not be expressed in the linear form where stiffness matrix is independent of $\boldsymbol{r}_{u}$. The numerical method must be utilized. Using the iterative Newton method, we can express next iterative step $m$ by the following identity

$$
{ }^{m+1} \boldsymbol{r}_{u}={ }^{m} \boldsymbol{r}_{u}+\delta^{m} \boldsymbol{r}_{u},
$$

where the increment $\delta^{m} \boldsymbol{r}_{u}$ equals to

$$
\delta^{m} \boldsymbol{r}_{u}=\left(\mathbf{K}_{u}^{N L}\right)^{-1}\left(\boldsymbol{R}\left({ }^{m} \boldsymbol{r}_{u}\right)-\boldsymbol{R}_{s}\right),
$$

and $\mathbf{K}_{u}^{N L}$ represents the tangential matrix and bracket $\left(\boldsymbol{R}\left({ }^{m} \boldsymbol{r}_{u}\right)-\boldsymbol{R}_{s}\right)$ represents the vector of unbalanced forces. The tangential matrix can be obtained as the Hessian matrix of functional $\Psi$. In discrete form it reads

$$
\mathbf{K}_{u}^{N L}=\left((1-\eta)\left(\boldsymbol{r}_{s}^{e}\right)^{\mathrm{T}} \mathbf{M}_{2} \boldsymbol{r}_{s}^{e}+\eta\right) \frac{\partial^{2} \Psi^{+}\left({ }^{m} \boldsymbol{r}_{u}^{e}\right)}{\left(\partial^{m} \boldsymbol{r}_{u}^{e}\right)^{2}}+\frac{\partial^{2} \Psi^{-}\left({ }^{m} \boldsymbol{r}_{u}^{e}\right)}{\left(\partial^{m} \boldsymbol{r}_{u}^{e}\right)^{2}}
$$

The discrete form (19) remains unchanged and iteration process is still valid. However each vector ${ }^{k} \boldsymbol{r}_{u}$ is obtained through an inner iteration process described by the equation (23).

\section{Conclusion}

The paper introduces variational based damage phase-field formulation for beams and plates and extends it for large deflection beams. Von Kármán term is employed in strain equation for beams. Consequently, inner iteration process for displacement field is needed. Update of damage field remains without changes.

\section{Acknowledgments}

This publication was supported by the Czech Science Foundation, the grant No. 16-14770S.

\section{References}

Kiendl, J., Ambati, M., De Lorenzis, L., Gomez, H., Reali, A. (2016), Phase-field description of brittle fracture in plates and shells. Computer Methods in Applied Mechanics and Engineering, Vol 312, pp 374-394.

Miehe, C., Hofacker, M., Welschinger, F. (2010), A phase field model for rate-independent crack propagation: Robust algorithmic implementation based on operator splits. Computer Methods in Applied Mechanics and Engineering, Vol 199, No. 45, pp 2765-2778.

Vignollet, J., May, S., de Borst, R., Verhoosel, C. V. (2014), Phase-field models for brittle and cohesive fracture. Meccanica, Vol 49, No. 11, pp 2587-2601. 